\title{
\begin{tabular}{l|l} 
pcori $).$ & PATIENT-CENTERED OUTCOMES RESEARCH INSTITUTE \\
RESEARCH SUMMARY
\end{tabular}
}

\section{Comparing Results of Three Treatments for Idiopathic Subglottic Stenosis}

Principal investigator

Alexander Gelbard, MD
Organization

Vanderbilt University Medical Center

\section{What was the research about?}

Idiopathic subglottic stenosis, or iSGS, is a rare disease that is most common among white women. With this disease, the trachea, or windpipe, narrows for unknown reasons. iSGS may affect quality of life by making it difficult for patients to breathe and by limiting daily activities such as talking and swallowing. iSGS can be treated but may come back after treatment. Having information about how different treatments for iSGS compare with each other can help patients and their doctors choose the treatment that works best for the patients.

In this study, the research team compared three common treatments for iSGS:

- Endoscopic dilation. Doctors go through the patient's mouth and use a balloon or other tool to stretch open the trachea.

- Endoscopic resection with long-term medicine use. Doctors go through the patient's mouth and use a laser to remove the blockage in the trachea. After the treatment, patients require long-term medicine use.

- Cricotracheal resection. Doctors cut open the front of the neck to remove the blockage in the trachea and then rebuild the airway.

Patients decided, with their doctors, which of the three treatments to get. Following treatment, the research team tracked symptoms to see if and how quickly iSGS symptoms came back.

\section{What were the results?}

Within three years of treatment, 23 percent of patients needed another treatment because symptoms of iSGS came back. Patients who received cricotracheal resection had the lowest rate of needing more treatments. Additional treatments were needed in

- 1 percent of patients who received cricotracheal resection

- 12 percent of patients who received endoscopic resection with long-term medicine use

- 28 percent of patients who received endoscopic dilation

After one year

- Patients who received endoscopic dilation reported worse breathing ability and quality of life than patients who received the other two treatments.

- Patients who received a cricotracheal resection reported worse speaking ability than patients who received the other two treatments.

- Swallowing ability was similar for the three treatments.

\section{Who was in the study?}

This study included 810 patients from 40 clinics in the United States, Australia, France, Iceland, Norway, and the United Kingdom. Of these patients, 97 percent were white. The median age was 50, and 98 percent 
were women. Before the study, patients hadn't received treatment for iSGS.

\section{What did the research team do?}

The research team looked at patients' medical records and surveyed patients for three years. Every three months, the team looked at whether patients needed another treatment. Every six months, the team asked patients about quality of life and problems with breathing, speaking, and swallowing.

Patients with iSGS and clinicians treating iSGS were members of the research team and were involved throughout the study.

\section{What were the limits of the study?}

The research team didn't assign patients to a treatment by chance. Therefore, the team can't be sure that the results were from the treatments or were caused by something the team didn't study. For example, some doctors might be better trained in one treatment than another, which could affect how well patients do after treatment.

Future studies could track patients for longer than three years to learn if the treatment effects continue over time.

\section{How can people use the results?}

Doctors and patients can use the results when considering treatment decisions for iSGS.

To learn more about this project, visit www.pcori.org/Gelbard233. 